\title{
SHARP SINGULAR ADAMS INEQUALITIES IN HIGH ORDER SOBOLEV SPACES*
}

\author{
NGUYEN LAM ${ }^{\dagger}$ AND GUOZHEN LU
}

\begin{abstract}
In this paper, we prove a version of weighted inequalities of exponential type for fractional integrals with sharp constants in any domain of finite measure in $\mathbb{R}^{n}$. Using this we prove a sharp singular Adams inequality in high order Sobolev spaces in bounded domain at critical case. Then we prove sharp singular Adams inequalities for high order derivatives on unbounded domains. Our results extend the singular Moser-Trudinger inequalities of first order in $[7,46,39,11]$ to the higher order Sobolev spaces $W^{m, \frac{n}{m}}$ and the results of [47] on Adams type inequalities in unbounded domains to singular case. We also prove a sharp singular Adams inequality on $W^{2,2}\left(\mathbb{R}^{4}\right)$ with standard Sobolev norm at the critical case.
\end{abstract}

Key words. Moser-Trudinger inequalities, Adams type inequalities, singular Adams inequalities, fractional integrals.

AMS subject classifications. 42B37, 42B35, 35J62, 26 D60.

1. Introduction. Let $\Omega \subset \mathbb{R}^{n}, n \geq 2$ be a smooth bounded domain, and $W_{0}^{1, n}(\Omega)$ be the completion of $C_{0}^{\infty}(\Omega)$ under the norm $\|u\|_{W_{0}^{1, n}(\Omega)}=$ $\left[\int_{\Omega}\left(|u|^{n}+|\nabla u|^{n}\right) d x\right]^{1 / n}$. The classical Moser-Trudinger inequality $[42,45,55,57]$ which plays an important role in analysis says that

$$
\sup _{u \in W_{0}^{1, n}(\Omega),\|\nabla u\|_{n} \leq 1} \frac{1}{|\Omega|} \int_{\Omega} \exp \left(\beta|u|^{\frac{n}{n-1}}\right) d x<+\infty
$$

for any $\beta \leq \beta_{n}=n \omega_{n-1}^{\frac{1}{n-1}}$, where $\omega_{n-1}=\frac{2 \pi^{\frac{n}{2}}}{\Gamma\left(\frac{n}{2}\right)}$ is the area of the surface of the unit $n$-ball. Moreover, this constant $\beta_{n}$ is sharp in the sense that if $\beta>\beta_{n}$, then supremum is infinite. Here and in the sequel, for any real number $p>1,\|\cdot\|_{p}$ denotes the $L^{p}$-norm with respect to the Lebesgue measure.

There is also another well known inequality in analysis: the Hardy inequality. Thus it is very natural to establish an interpolation of Hardy inequality and MoserTrudinger inequality. Inspired by the following Hardy inequality [4]:

$$
\left(\frac{n-1}{n}\right)^{n} \int_{\Omega} \frac{|u|^{n}}{|x|^{n}\left(\log \frac{R}{|x|}\right)^{n}} d x \leq \int_{\Omega}|\nabla u|^{n}
$$

where $u \in W_{0}^{1, n}(\Omega)$ and $R \geq \sup _{\Omega}\left(|x| e^{2 / n}\right)$, Adimurthi and Sandeep proved in [7] a singular Moser-Trudinger inequality with the sharp constant:

Theorem A. Let $\Omega$ be an open and bounded set in $\mathbb{R}^{n}$. There exists a constant $C_{0}=C_{0}(n,|\Omega|)>0$ such that

$$
\int_{\Omega} \frac{\exp \left(\beta|u|^{\frac{n}{n-1}}\right)}{|x|^{\alpha}} d x \leq C_{0}
$$

*Received April 1, 2012; accepted for publication November 29, 2012. Research is partly supported by a US NSF grant DMS0901761.

${ }^{\dagger}$ Department of Mathematics, Wayne State University, Detroit, MI 48202, USA (nguyenlam@ wayne.edu).

${ }^{\ddagger}$ Corresponding Author. Department of Mathematics, Wayne State University, Detroit, MI 48202, USA (gzlu@math.wayne.edu). 
for any $\alpha \in[0, n), 0 \leq \beta \leq\left(1-\frac{\alpha}{n}\right) \beta_{n}$, any $u \in W_{0}^{1, n}(\Omega)$ with $\int_{\Omega}|\nabla u|^{n} d x \leq 1$. Moreover, this constant $\left(1-\frac{\alpha}{n}\right) \beta_{n}$ is sharp in the sense that if $\beta>\left(1-\frac{\alpha}{n}\right) \beta_{n}$, then the above inequality can no longer hold with some $C_{0}$ independent of $u$.

There is another improved Moser-Trudinger inequality on the disk $B \subset \mathbb{R}^{2}$, which was recently proved and studied in [10, 41]:

$$
\sup _{u \in W_{0}^{1,2}(B),\|\nabla u\|_{2} \leq 1} \int_{B} \frac{\exp \left(4 \pi|u|^{2}\right)-1}{\left(1-|x|^{2}\right)^{2}} d x<+\infty .
$$

Very recently, Wang and Ye [56] proved an interesting Hardy-Moser-Trudinger inequality on the unit disk in $\mathbb{R}^{2}$, which improves the classical Moser-Trudinger inequality and the classical Hardy inequality at the same time. Namely, there exists a constant $C_{0}>0$ such that

$$
\int_{B} e^{\frac{4 \pi u^{2}}{H(u)}} d x \leq C_{0}<\infty, \forall u \in C_{0}^{\infty}(B) \backslash\{0\}
$$

where

$$
H(u)=\int_{B}|\nabla u|^{2} d x-\int_{B} \frac{u^{2}}{\left(1-|x|^{2}\right)^{2}} d x
$$

We also mention results of sharp Moser-Trudinger trace inequalities and sharp Moser-Trudinger inequalities without boundary conditions by Cianchi $[17,18]$ and the sharp Moser-Trudinger type inequalities for the Hessian equation by Tian and Wang [53].

We notice that when $\Omega$ has infinite volume, the usual Moser-Trudinger inequalities become meaningless. In the case $|\Omega|=+\infty$, the following modified Moser-Trudinger type inequality can be established:

Theorem B. For all $\beta>0,0 \leq \alpha<n$ and $u \in W^{1, n}\left(\mathbb{R}^{n}\right)(n \geq 2)$, there holds

$$
\int_{\mathbb{R}^{n}} \frac{\phi\left(\beta|u|^{\frac{n}{n-1}}\right)}{|x|^{\alpha}} d x<\infty
$$

Furthermore, we have for all $\beta \leq\left(1-\frac{\alpha}{n}\right) \beta_{n}$ and $\tau>0$,

$$
\sup _{\|u\|_{1, \tau} \leq 1} \int_{\mathbb{R}^{n}} \frac{\phi\left(\beta|u|^{\frac{n}{n-1}}\right)}{|x|^{\alpha}} d x<\infty
$$

where

$$
\begin{aligned}
\phi(t) & =e^{t}-\sum_{j=0}^{n-2} \frac{t^{j}}{j !} \\
\|u\|_{1, \tau} & =\left(\int_{\mathbb{R}^{n}}\left(|\nabla u|^{n}+\tau|u|^{n}\right) d x\right)^{1 / n} .
\end{aligned}
$$


Moreover, this constant $\left(1-\frac{\alpha}{n}\right) \beta_{n}$ is sharp in the sense that if $\beta>\left(1-\frac{\alpha}{n}\right) \beta_{n}$, then the supremum is infinity.

The above modified Moser-Trudinger type inequality when $\alpha=0$ was established by B. Ruf [46] in dimension two and Y.X. Li and Ruf [39] in general dimension. It was then extended to the singular case $0 \leq \alpha<n$ by Adimurthi and Yang [11]. Indeed, such type of inequality on unbounded domains in the subcritical case $\beta<\beta_{n}(\alpha=0)$ was first established by D. Cao [15] in dimension two and by Adachi and Tanaka [1] in high dimension.

In the case of compactly supported functions, D. Adams [2] extended the original Moser-Trudinger inequality to the higher order space $W_{0}^{m, \frac{n}{m}}(\Omega)$. In fact, Adams proved the following inequality:

Theorem C. There exists a constant $C_{0}=C(n, m)>0$ such that for any $u \in W_{0}^{m, \frac{n}{m}}(\Omega)$ and $\left\|\nabla^{m} u\right\|_{L^{\frac{n}{m}(\Omega)}} \leq 1$, then

$$
\frac{1}{|\Omega|} \int_{\Omega} \exp \left(\beta|u(x)|^{\frac{n}{n-m}}\right) d x \leq C_{0}
$$

for all $\beta \leq \beta(n, m)$ where

$$
\beta(n, m)=\left\{\begin{array}{ll}
\frac{n}{w_{n-1}}\left[\frac{\pi^{n / 2} 2^{m} \Gamma\left(\frac{m+1}{2}\right)}{\Gamma\left(\frac{n-m+1}{2}\right)}\right]_{n}^{\frac{n}{n-m}} \text { when } m \text { is odd } \\
\frac{n}{w_{n-1}}\left[\frac{\pi^{n / 2} 2^{m} \Gamma\left(\frac{m}{2}\right)}{\Gamma\left(\frac{n-m}{2}\right)}\right]^{\frac{n}{n-m}} \text { when } m \text { is even }
\end{array} .\right.
$$

Furthermore, for any $\beta>\beta(n, m)$, the integral can be made as large as possible.

Note that $\beta(n, 1)$ coincides with Moser's value of $\beta_{n}$ and $\beta(2 m, m)=2^{2 m} \pi^{m} \Gamma(m+$ 1) for both odd and even $m$. Here, we use the symbol $\nabla^{m} u$, where $m$ is a positive integer, to denote the $m$-th order gradient for $u \in C^{m}$, the class of $m$-th order differentiable functions:

$$
\nabla^{m} u=\left\{\begin{array}{ll}
\triangle^{\frac{m}{2}} u & \text { for } m \text { even } \\
\nabla \triangle^{\frac{m-1}{2}} u \text { for } m \text { odd }
\end{array} .\right.
$$

where $\nabla$ is the usual gradient operator and $\triangle$ is the Laplacian. We use $\left\|\nabla^{m} u\right\|_{p}$ to denote the $L^{p}$ norm $(1 \leq p \leq \infty)$ of the function $\left|\nabla^{m} u\right|$, the usual Euclidean length of the vector $\nabla^{m} u$. We also use $W_{0}^{k, p}(\Omega)$ to denote the Sobolev space which is a completion of $C_{0}^{\infty}(\Omega)$ under the norm of $\left(\sum_{j=0}^{k}\left\|\nabla^{j} u\right\|_{L^{p}(\Omega)}^{p}\right)^{1 / p}$.

Recently, in the setting of the Sobolev space with homogeneous Navier boundary conditions $W_{N}^{m, \frac{n}{m}}(\Omega)$ :

$$
W_{N}^{m, \frac{n}{m}}(\Omega):=\left\{u \in W^{m, \frac{n}{m}}: \Delta^{j} u=0 \text { on } \partial \Omega \text { for } 0 \leq j \leq\left[\frac{m-1}{2}\right]\right\}
$$

the Adams inequality was extended by Tarsi [52]. Note that $W_{N}^{m, \frac{n}{m}}(\Omega)$ contains the Sobolev space $W_{0}^{m, \frac{n}{m}}(\Omega)$ as a closed subspace. 
The Adams type inequality on Sobolev spaces $W_{0}^{m, \frac{n}{m}}(\Omega)$ when $\Omega$ has infinite volume and $m$ is an even integer was studied recently by Ruf and Sani [47]. In fact, they proved the following

THEOREM D. If $m$ is an even integer less than $n$, then there exists a constant $C_{m, n}>0$ such that for any domain $\Omega \subseteq \mathbb{R}^{n}$

$$
\sup _{u \in W_{0}^{m, \frac{n}{m}}(\Omega),\|u\|_{m, n} \leq 1} \int_{\Omega} \phi\left(\beta_{0}(n, m)|u|^{\frac{n}{n-m}}\right) d x \leq C_{m, n}
$$

where

$$
\begin{aligned}
\beta_{0}(n, m) & =\frac{n}{\omega_{n-1}}\left[\frac{\pi^{\frac{n}{2}} 2^{m} \Gamma\left(\frac{m}{2}\right)}{\Gamma\left(\frac{n-m}{2}\right)}\right]^{\frac{n}{n-m}}, \\
\phi(t) & =e^{t}-\sum_{j=0}^{j \frac{n}{m}-2} \frac{t^{j}}{j !} \\
j_{\frac{n}{m}} & =\min \left\{j \in \mathbb{N}: j \geq \frac{n}{m}\right\} \geq \frac{n}{m} .
\end{aligned}
$$

Moreover, this inequality is sharp in the sense that if we replace $\beta_{0}(n, m)$ by any larger $\beta$, then the above supremum will be infinity.

In the above result, Ruf and Sani used the norm

$$
\|u\|_{m, n}=\left\|(-\Delta+I)^{\frac{m}{2}} u\right\|_{\frac{n}{m}}
$$

which is equivalent to the standard Sobolev norm

$$
\|u\|_{W^{m, \frac{n}{m}}}=\left(\|u\|_{\frac{n}{m}}^{\frac{n}{m}}+\sum_{j=1}^{m}\left\|\nabla^{j} u\right\|_{\frac{n}{m}}^{\frac{n}{m}}\right)^{\frac{m}{n}}
$$

In particular, if $u \in W_{0}^{m, \frac{n}{m}}(\Omega)$ or $u \in W^{m, \frac{n}{m}}\left(\mathbb{R}^{n}\right)$, then $\|u\|_{W^{m, \frac{n}{m}}} \leq\|u\|_{m, n}$.

Because the result of Ruf and Sani [47] only treats the case when $m$ is even, thus it leaves an open question if Ruf and Sani's theorem still holds when $m$ is odd. Recently, the authors of [29] have established the results of Adams type inequalities on unbounded domains when $m$ is odd. More precisely, the first result of [29] is as follows:

TheOREM E. Let $m$ be an odd integer less than $n: m=2 k+1, k \in \mathbb{N}$. There holds

$$
\sup _{u \in W^{m, \frac{n}{m}}\left(\mathbb{R}^{n}\right),\left\|\nabla(-\Delta+I)^{k} u\right\|_{\frac{n}{m}}^{\frac{n}{m}}+\left\|(-\Delta+I)^{k} u\right\|_{\frac{n}{m}}^{\frac{n}{m}} \leq 1} \int_{\mathbb{R}^{n}} \phi\left(\beta(n, m)|u|^{\frac{n}{n-m}}\right) d x<\infty .
$$

Moreover, the constant $\beta(n, m)$ is sharp.

In the special case $n=2 m$, we have the following stronger results in [29]:

Theorem F. Let $m=2 k+1, k \in \mathbb{N}$. For all $\tau>0$, there holds

$$
\sup _{u \in W^{m, 2}\left(\mathbb{R}^{2 m}\right),\left\|\nabla(-\Delta+\tau I)^{k} u\right\|_{2}^{2}+\tau\left\|(-\Delta+\tau I)^{k} u\right\|_{2}^{2} \leq 1} \int_{\mathbb{R}^{2 m}}\left(e^{\beta(2 m, m) u^{2}}-1\right) d x<\infty .
$$


Moreover, the constant $\beta(2 m, m)$ is sharp in the sense that if we replace $\beta(2 m, m)$ by any $\beta>\beta(2 m, m)$, then the supremum is infinity.

The result of [47] (stated as Theorem D above) for $m$ being even were also extended recently using the standard Sobolev norm by Yang in the special case $n=4$ and $m=2$ [58] and by the authors [29] to the case $n=2 m$ for all $m$ being both odd and even. More precisely, the following has been established by the authors in [29]:

TheOREM G. Let $m \geq 2$ be an integer. For all constants $a_{0}=1, a_{1}, \ldots, a_{m}>0$, there holds

$$
\sup _{u \in W^{m, 2}\left(\mathbb{R}^{2 m}\right), \int_{\mathbb{R}^{2 m}}\left(\sum_{j=0}^{m} a_{m-j}\left|\nabla^{j} u\right|^{2}\right) d x \leq 1} \int_{\mathbb{R}^{2 m}}\left[\exp \left(\beta(2 m, m)|u|^{2}\right)-1\right] d x<\infty .
$$

Furthermore this inequality is sharp, i.e., if $\beta(2 m, m)$ is replaced by any $\beta>$ $\beta(2 m, m)$, then the supremum is infinite.

As a corollary of the above theorem, we have the following Adams type inequality with the standard Sobolev norm:

TheOREM H. Let $m \geq 1$ be an integer. There holds

$$
\sup _{u \in W^{m, 2}\left(\mathbb{R}^{2 m}\right),\|u\|_{W^{m, 2}} \leq 1} \int_{\mathbb{R}^{2 m}}\left[\exp \left(\beta(2 m, m)|u|^{2}\right)-1\right] d x<\infty .
$$

Furthermore this inequality is sharp, i.e., if $\beta(2 m, m)$ is replaced by any $\alpha>$ $\beta(2 m, m)$, then the supremum is infinite.

Moser-Trudinger type inequalities and Adams type inequalities have important applications in geometric analysis and partial differential equations, especially in the study of the exponential growth partial differential equations where the nonlinear term behaves like $e^{\alpha|u|^{\frac{n}{n-m}}}$ as $|u| \rightarrow \infty$. There has been a vast literature in this direction. We refer the interested reader to [13], [16], [3], [7], [6], [9], [11], [8], [20], [21], [19], $[27,28],[49],[50]$ and the references therein. When the nonlinear terms do not satisfy the Ambrosetti-Rabinowitz condition, existence of nonnegative and nontrivial weak solutions has also been verified in [30] and [32]. See also a survey article [31].

In this paper, we will first establish a sharp inequality of exponential type with weights $\frac{1}{|x|^{\alpha}}$ for the fractional integrals.

THEOREM 1.1. Let $1<p<\infty, 0 \leq \alpha<n$ and $\Omega \subset \mathbb{R}^{n}$ be an open set with $|\Omega|<\infty$. Then there is a constant $c_{0}=c_{0}(p, \Omega)$ such that for all $f \in L^{p}\left(\mathbb{R}^{n}\right)$ with support contained in $\Omega$,

$$
\int_{\Omega} \frac{\exp \left(\left(1-\frac{\alpha}{n}\right) \frac{n}{\omega_{n-1}}\left|\frac{I_{\gamma} * f(x)}{\|f\|_{p}}\right|^{p^{\prime}}\right)}{|x|^{\alpha}} d x \leq c_{0},
$$

where $\gamma=n / p$ and $I_{\gamma} * f(x)=\int|x-y|^{\gamma-n} f(y) d y$ is the Riesz potential of order $\gamma$.

We will prove the above theorem by using the approach of Adams in the non weighted case (i.e., $\alpha=0$ ). It can also be deduced from a general result in [23] by verifying our weight satisfies the condition in [23]. 
Next, we will establish a version of singular Adams inequality on bounded domains. More precisely, we will prove that:

THEOREM 1.2. Let $0 \leq \alpha<n$ and $\Omega$ be a bounded domain in $\mathbb{R}^{n}$. Then for all $0 \leq \beta \leq \beta_{\alpha, n, m}=\left(1-\frac{\alpha}{n}\right) \bar{\beta}(n, m)$, we have

$$
\sup _{u \in W_{0}^{m, \frac{n}{m}}(\Omega),\left\|\nabla^{m} u\right\|_{\frac{n}{m}} \leq 1} \int_{\Omega} \frac{e^{\beta|u|^{\frac{n}{n-m}}}}{|x|^{\alpha}} d x<\infty .
$$

When $\beta>\beta_{\alpha, n, m}$, the supremum is infinite. Moreover, when $m$ is an even number, the Sobolev space $W_{0}^{m, \frac{n}{m}}(\Omega)$ in the above supremum can be replaced by a larger Sobolev space $W_{N}^{m, \frac{n}{m}}(\Omega)$.

A similar singular Adams inequality on the Heisenberg group has been established in [35]. Using the above Theorem 1.2, we will then set up the singular Adams inequality for the space $W^{m, \frac{n}{m}}\left(\mathbb{R}^{n}\right)$ when $m$ is an even integer number:

TheOREM 1.3. Let $0 \leq \alpha<n, m>0$ be an even integer less than $n$. Then for all $0 \leq \beta \leq \beta_{\alpha, n, m}=\left(1-\frac{\alpha}{n}\right) \beta_{0}(n, m)$, we have

$$
\sup _{u \in W^{m, \frac{n}{m}}\left(\mathbb{R}^{n}\right),\left\|(-\Delta+I)^{\frac{m}{2}} u\right\|_{\frac{n}{m}} \leq 1} \int_{\mathbb{R}^{n}} \frac{\phi\left(\beta|u|^{\frac{n}{n-m}}\right) d x}{|x|^{\alpha}} d x<\infty
$$

where $\phi(t)=e^{t}-\sum_{j=0}^{j \frac{n}{m}-2} \frac{t^{j}}{j !}$. Moreover, when $\beta>\beta_{\alpha, n, m}$, the supremum is infinite.

Finally, in the special case $n=2 m=4$, we will prove a singular Adams inequality in the spirit of Theorem $\mathrm{G}$ above.

TheOrem 1.4. Let $0 \leq \alpha<4$. Assume that $\tau>0$ and $\sigma>0$ are any two positive constants. Then for all $0 \leq \beta \leq \beta_{\alpha}=\left(1-\frac{\alpha}{4}\right) 32 \pi^{2}$, we have

$$
\sup _{u \in W^{2,2}\left(\mathbb{R}^{4}\right), \int_{\mathbb{R}^{4}}\left(|\Delta u|^{2}+\tau|\nabla u|^{2}+\sigma|u|^{2}\right) \leq 1} \int_{\mathbb{R}^{4}} \frac{\left(e^{\beta u^{2}}-1\right)}{|x|^{\alpha}} d x<\infty .
$$

Moreover, when $\beta>\beta_{\alpha}$, the supremum is infinite.

As we can see, when $\alpha=0$, this theorem is already included in Theorem G. When $0<\alpha<4$, we note that the above inequality (1.3) for the subcritical case $\beta<\beta_{\alpha}=$ $\left(1-\frac{\alpha}{4}\right) 32 \pi^{2}$ was proved in [58]. However, the critical case $\beta=\left(1-\frac{\alpha}{4}\right) 32 \pi^{2}$ is much harder to prove. Thus, our Theorem 1.4 in the critical case settles a unsolved question remained in [58].

Recently, in [33] we developed a new method of rearrangement-free to establish the sharp Adams inequalities on high order Sobolev spaces $W^{\alpha, \frac{n}{\alpha}}\left(\mathbb{R}^{n}\right)$ of any fractional order $0<\alpha<n$. The approach used in [33] is completely different from and substantially simpler than those in [47], [29] and this paper. We completely avoid the symmetrization argument and we do not use the complicated comparison principle for solutions to the polyharmonic operators unlike as used in [47], [29] and this paper. This method can be adapted to many other settings such as Rieamannian 
manifolds and metric measure spaces where no symmetrization is available. In fact, sharp Moser-Trudinger inequalities on the Heisenberg group on the entire Heisenberg group has been established in [34].

More precisely, we have proved in [33]:

THEOREM I Let $0<\alpha<n$ be an arbitrary real positive number, $p=\frac{n}{\alpha}$ and $\tau>0$. There holds

$$
\sup _{u \in W^{\alpha, p}\left(\mathbb{R}^{n}\right),\left\|(\tau I-\Delta)^{\frac{\alpha}{2}} u\right\|_{p} \leq 1} \int_{\mathbb{R}^{n}} \phi_{n, \alpha}\left(\beta_{0}(n, \alpha)|u|^{p^{\prime}}\right) d x<\infty
$$

where

$$
\beta_{0}(n, \alpha)=\frac{n}{\omega_{n-1}}\left[\frac{\pi^{n / 2} 2^{\alpha} \Gamma(\alpha / 2)}{\Gamma\left(\frac{n-\alpha}{2}\right)}\right]^{p^{\prime}} .
$$

Furthermore this inequality is sharp, i.e., if $\beta_{0}(n, \alpha)$ is replaced by any $\gamma>\beta_{0}(n, \alpha)$, then the supremum is infinite. We have used the notations

$$
\begin{aligned}
\phi_{n, \alpha}(t) & =e^{t}-\sum_{j=0}^{j_{\frac{n}{m}}-2} \frac{t^{j}}{j !} \\
j_{\frac{n}{\alpha}} & =\min \left\{j \in \mathbb{N}: j \geq \frac{n}{\alpha}\right\} \geq \frac{n}{\alpha} .
\end{aligned}
$$

Moreover, we have recently improved in [33] our Theorem 1.4. We established in [33] an improved version of the Adams type inequality in the Sobolev space $W^{2, m}\left(\mathbb{R}^{2 m}\right)$ for any $m$. In this special case, it has been proved in [33] that:

Theorem J. Let $0 \leq \alpha<2 m$ and $\tau>0$. Then for all $0 \leq \beta \leq$ $\left(1-\frac{\alpha}{2 m}\right) \beta(2 m, 2)$, we have

$$
\sup _{u \in W^{2, m}\left(\mathbb{R}^{2 m}\right),} \sup _{\mathbb{R}^{2 m}|\Delta u|^{m}+\tau|u|^{m} \leq 1} \int_{\mathbb{R}^{2 m}} \frac{\phi_{2 m, 2}\left(\beta|u|^{\frac{m}{m-1}}\right)}{|x|^{\alpha}} d x<\infty .
$$

Moreover, the constant $\left(1-\frac{\alpha}{2 m}\right) \beta(2 m, 2)$ is sharp in the sense that if $\beta>$ $\left(1-\frac{\alpha}{2 m}\right) \beta(2 m, 2)$, then the supremun is infinite.

We should note this result does not require the restriction on the full standard norm and hence, it extends Theorem 1.4 here. Indeed, the results stated in Theorem 1.4 are for the special case $m=2$ and they require that the full standard norm $\int_{\mathbb{R}^{4}}\left(|\Delta u|^{2}+\sigma|\nabla u|^{2}+\tau|u|^{2}\right) d x$ is less than 1.

We remark that we have also established in [37] sharp affine Moser-Trudinger inequalities and improved Moser-Trudinger-Adams inequalities in the spirit of [38], [5], [40]. In particular, among many sharpened versions of Adams inequalities, we proved in [37] the following:

Theorem K. Let $0 \leq \beta<2 m$ and $\tau>0$. Then there exists a constant $C=$ $C(m, \beta)>0$ such that for all $u \in C_{0}^{\infty}\left(\mathbb{R}^{2 m}\right) \backslash\{0\},\|\Delta u\|_{m}<1$, we have

$$
\int_{\mathbb{R}^{2 m}} \frac{\phi_{2 m, 2}\left(\frac{2^{\frac{1}{m-1}}\left(1-\frac{\beta}{2 m}\right) \beta(2 m, 2)}{\left(1+\|\Delta u\|_{m}^{m}\right)^{\frac{1}{m-1}}}|u|^{\frac{m}{m-1}}\right)}{|x|^{\beta}} d x \leq C(m, \beta) \frac{\|u\|_{m}^{m-\frac{\beta}{2}}}{\left|1-\|\Delta u\|_{m}^{m}\right|^{1-\frac{\beta}{2 m}}} .
$$


Consequently, we have that there exists a constant $C=C(m, \beta, \tau)>0$ such that

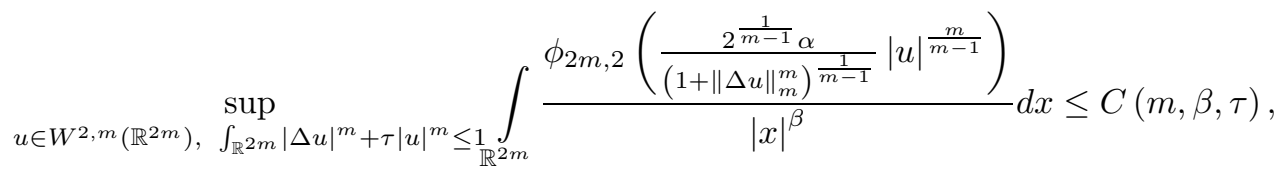

for all $0 \leq \alpha \leq\left(1-\frac{\beta}{2 m}\right) \beta(2 m, 2)$. When $\alpha>\left(1-\frac{\beta}{2 m}\right) \beta(2 m, 2)$, the supremum is infinite.

Concerning the Adams inequality for unbounded domains, in the spirit of AdachiTanaka [1], T. Ogawa and T. Ozawa [43] in the case $\frac{n}{m}=2$ and T. Ozawa [44] in the general case proved that there exist positive constants $\alpha$ and $C_{\alpha}$ such that

$$
\int_{\mathbb{R}^{n}} \phi_{n, m}\left(\alpha|u|^{\frac{n}{n-m}}\right) d x \leq C_{\alpha}\|u\|_{\frac{n}{m}}^{\frac{n}{m}}, \forall u \in W^{m, \frac{n}{m}}\left(\mathbb{R}^{n}\right),\left\|\nabla^{m} u\right\|_{\frac{n}{m}} \leq 1 .
$$

Their approach of proving the above result is similar to the idea of Yudovich [57], Pohozaev [45] and Trudinger [55], namely using the sum up of power series, and thus the problem of determining the best constant cannot be investigated by this way. In fact, as pointed out in [48], it is still left as an open problem. Thus, it is very interesting to identify the best constants in such inequalities.

Recently, in [37], the authors establish the sharp subcritical Adams type inequalities in some special cases. More precisely, we have proved in [37] that

Theorem L. For any $\alpha \in(0, \beta(n, 2))$, there exists a constant $C_{\alpha}>0$ such that

$$
\int_{\mathbb{R}^{n}} \phi_{n, 2}\left(\alpha|u|^{\frac{n}{n-2}}\right) d x \leq C_{\alpha}\|u\|_{\frac{n}{2}}^{\frac{n}{2}}, \forall u \in W^{2, \frac{n}{2}}\left(\mathbb{R}^{n}\right),\|\Delta u\|_{\frac{n}{2}} \leq 1 .
$$

Theorem M. For any $\alpha \in(0, \beta(2 m, m))$, there exists a constant $C_{\alpha}>0$ such that

$$
\int_{\mathbb{R}^{2 m}} \phi_{2 m, m}\left(\alpha|u|^{2}\right) d x \leq C_{\alpha}\|u\|_{2}^{2}, \forall u \in W^{m, 2}\left(\mathbb{R}^{2 m}\right),\left\|\nabla^{m} u\right\|_{2} \leq 1 .
$$

It was proved in [26] that the inequality (1.4) in Theorem L does not hold when $\alpha>\beta(n, 2)$, neither does inequality (1.5) in Theorem M when $\alpha>\beta(2 m, m)$. Nevertheless, we still cannot verify the borderline case $\alpha=\beta(n, 2)$ in Theorem $\mathrm{L}$ and $\alpha=\beta(2 m, m)$ in Theorem M. These are still left as open problems in [37]. We should mention that using the rearrangement-free argument developed in [33], we have proved in [36] sharp subcritical Moser-Trudinger type inequalities on the Heisenberg group and sharp Moser-Trudinger inequality on the Heisenberg group with full norm in [34].

Our paper is organized as follows: In Section 2, we give some preliminaries. Section 3 deals with the sharp weighted inequality of exponential type for fractional integrals (Theorem 1.1). The singular Adams inequality for the bounded domains (Theorem 1.2) will be proved in Section 4. Theorem 1.2 will be used to prove Theorem 1.3 and Theorem 1.4 in Section 5. 
2. Some preliminaries. In this section, we provide some preliminaries. For $u \in W^{m, p}(\Omega)$ with $1 \leq p<\infty$, we will denote by $\nabla^{j} u, j \in\{1,2, \ldots, m\}$, the $j-t h$ order gradient of $u$, namely

$$
\nabla^{j} u=\left\{\begin{array}{ll}
\triangle^{\frac{j}{2}} u & \text { for } j \text { even } \\
\nabla \triangle^{\frac{j-1}{2}} u \text { for } j \text { odd }
\end{array} .\right.
$$

We now introduce the Sobolev space of functions with homogeneous Navier boundary conditions:

$$
W_{N}^{m, \frac{n}{m}}(\Omega):=\left\{u \in W^{m, \frac{n}{m}}(\Omega): \Delta^{j} u=0 \text { on } \partial \Omega \text { for } 0 \leq j \leq\left[\frac{m-1}{2}\right]\right\} .
$$

It is easy to see that $W_{N}^{m, \frac{n}{m}}(\Omega)$ contains $W_{0}^{m, \frac{n}{m}}(\Omega)$ as a closed subspace. We also define

$$
\begin{aligned}
& W_{\text {rad }}^{m, \frac{n}{m}}\left(B_{R}\right):=\left\{u \in W^{m, \frac{n}{m}}\left(B_{R}\right): u(x)=u(|x|) \text { a.e. in } B_{R}\right\}, \\
& W_{N, \text { rad }}^{m, \frac{n}{m}}\left(B_{R}\right)=W_{N}^{m, \frac{n}{m}}\left(B_{R}\right) \cap W_{\text {rad }}^{m, \frac{n}{m}}\left(B_{R}\right)
\end{aligned}
$$

where $B_{R}=\left\{x \in \mathbb{R}^{n}:|x|<R\right\}$ is a ball in $\mathbb{R}^{n}$.

Next, we will discuss the iterated comparison principle. Let $\Omega$ be a bounded domain in $\mathbb{R}^{n}$ and $B_{R}$ be an open ball with radius $R>0$ centered at 0 such that $|\Omega|=\left|B_{R}\right|$. Let $u: \Omega \rightarrow \mathbb{R}$ be a measurable function. The distribution function of $u$ is defined by

$$
\mu_{u}(t)=|\{x \in \Omega|| u(x) \mid>t\}| \forall t \geq 0 .
$$

The decreasing rearrangement of $u$ is defined by

$$
u^{*}(s)=\inf \left\{t \geq 0: \mu_{u}(t)<s\right\} \quad \forall s \in\left[0,\left|B_{R}\right|\right],
$$

and the spherically symmetric decreasing rearrangement of $u$ by

$$
\begin{aligned}
u^{\#}(x) & =u^{*}\left(\sigma_{n}|x|^{n}\right) \quad \forall x \in B_{R}, \\
\sigma_{n} & =\frac{\pi^{\frac{n}{2}}}{\Gamma\left(\frac{n}{2}+1\right)} \text { is the volume of the unit ball } B(0,1) \text { in } \mathbb{R}^{n} .
\end{aligned}
$$

We have that $u^{\#}$ is the unique nonnegative integrable function which is radially symmetric, nonincreasing and has the same distribution function as $|u|$.

Now, we introduce the Trombetti and Vazquez iterated comparision principle [54]: let $c>0$ and $u$ be a weak solution of

$$
\left\{\begin{array}{c}
-\Delta u+c u=f \text { in } B_{R} \\
u \in W_{0}^{1,2}\left(B_{R}\right)
\end{array}\right.
$$

where $f \in L^{\frac{2 n}{n+2}}\left(B_{R}\right)$. We have the following result that can be found in [54] (Inequality $(2.20))$ :

Proposition 2.1. If $u$ is a nonnegative weak solution of (2.1) then

$$
-\frac{d u^{*}}{d s}(s) \leq \frac{s^{\frac{2}{n}-2}}{n^{2} \sigma_{n}^{2 / n}} \int_{0}^{s}\left(f^{*}-c u^{*}\right) d t, \forall s \in\left(0,\left|B_{R}\right|\right) .
$$


Now, we consider the problem

$$
\left\{\begin{array}{c}
-\Delta v+c v=f^{\#} \text { in } B_{R} \\
v \in W_{0}^{1,2}\left(B_{R}\right)
\end{array}\right.
$$

Due to the radial symmetry of the equation, the unique solution $v$ of (2.3) is radially symmetric and we have

$$
-\frac{d \widehat{v}}{d s}(s)=\frac{s^{\frac{2}{n}-2}}{n^{2} \sigma_{n}^{2 / n}} \int_{0}^{s}\left(f^{*}-c \widehat{v}\right) d t, \forall s \in\left(0,\left|B_{R}\right|\right)
$$

where $\widehat{v}\left(\sigma_{n}|x|^{n}\right):=v(x)$. We have the following comparison of integrals in balls that again can be found in [54]:

Proposition 2.2. Let $u, v$ be weak solutions of (2.1) and (2.3) respectively. For every $r \in(0, R)$ we have

$$
\int_{B_{r}} u^{\#} d x \leq \int_{B_{r}} v d x
$$

and for every convex nondecreasing function $\phi:[0,+\infty) \rightarrow[0,+\infty)$ we have

$$
\int_{B_{r}} \phi(|u|) d x \leq \int_{B_{r}} \phi(|v|) d x .
$$

Next, we adapt the comparison principle to the polyharmonic operator. Let $u \in W^{m, 2}\left(B_{R}\right)$ be a weak solution of

$$
\left\{\begin{array}{c}
(-\Delta+c I)^{k} u=f \text { in } B_{R} \\
u \in W_{N}^{2 k, 2}\left(B_{R}\right)
\end{array}\right.
$$

where $m=2 k$ and $f \in L^{\frac{2 n}{n+2}}\left(B_{R}\right)$. If we consider the problem

$$
\left\{\begin{array}{c}
(-\Delta+c I)^{k} v=f^{\#} \text { in } B_{R} \\
v \in W_{N}^{2 k, 2}\left(B_{R}\right)
\end{array}\right.
$$

then we have the following comparison of integrals in balls:

Proposition 2.3. Let $u, v$ be weak solutions of the polyharmonic problems (2.5) and (2.6) respectively. Then for every $r \in(0, R)$ we have

$$
\int_{B_{r}} u^{\#} d x \leq \int_{B_{r}} v d x .
$$

Proof. The proof adapts the comparison principle as in [54] and [47]. We include a proof for its completeness. Since equations in (2.5) and (2.6) are considered with homogeneous Navier boundary conditions, they may be rewritten as second order systems:
$(P 1)\left\{\begin{array}{c}-\Delta u_{1}+c u_{1}=f \text { in } B_{R} \\ u_{1} \in W_{0}^{1,2}\left(B_{R}\right)\end{array}\right.$
$(P i)\left\{\begin{array}{c}-\Delta u_{i}+c u_{i}=u_{i-1} \text { in } B_{R} \\ u_{i} \in W_{0}^{1,2}\left(B_{R}\right)\end{array} \quad i \in\{2,3, \ldots, k\}\right.$
$(Q 1)\left\{\begin{array}{c}-\Delta v_{1}+c v_{1}=f^{\#} \text { in } B_{R} \\ v_{1} \in W_{0}^{1,2}\left(B_{R}\right)\end{array}\right.$
$(Q i)\left\{\begin{array}{c}-\Delta v_{i}+c v_{i}=v_{i-1} \text { in } B_{R} \\ v_{i} \in W_{0}^{1,2}\left(B_{R}\right)\end{array} \quad i \in\{2,3, \ldots, k\}\right.$ 
where $u_{k}=u$ and $v_{k}=v$. Thus we have to prove that for every $r \in(0, R)$

$$
\int_{B_{r}} u_{k}^{\#} d x \leq \int_{B_{r}} v_{k} d x
$$

By the above proposition (Proposition 2.2), we have

$$
\int_{B_{r}} u_{1}^{\#} d x \leq \int_{B_{r}} v_{1} d x
$$

Now, if we assume that

$$
\int_{B_{r}} u_{j}^{\#} d x \leq \int_{B_{r}} v_{j} d x \text { for all } j=1, \ldots, i
$$

we will prove that

$$
\int_{B_{r}} u_{i+1}^{\#} d x \leq \int_{B_{r}} v_{i+1} d x
$$

With no loss of generality, we may assume that $u_{i+1} \geq 0$. In fact, let $\bar{u}_{i+1}$ be a weak solution of

$$
\left\{\begin{array}{c}
-\Delta \bar{u}_{i+1}+c \bar{u}_{i+1}=\left|u_{i}\right| \text { in } B_{R} \\
\bar{u}_{i+1} \in W_{0}^{1,2}\left(B_{R}\right)
\end{array}\right.
$$

then the maximum principle implies that $\bar{u}_{i+1} \geq 0$ and $\bar{u}_{i+1} \geq\left|u_{i+1}\right|$ in $B_{R}$.

Since $u_{i+1}$ is a nonnegative weak solution of $(P(i+1))$ and $v_{i+1}$ is a nonnegative weak solution of $(Q(i+1))$, then by Proposition 2.1 we have

$$
\begin{aligned}
& -\frac{d u_{i+1}^{*}}{d s}(s) \leq \frac{s^{\frac{2}{n}-2}}{n^{2} \sigma_{n}^{2 / n}} \int_{0}^{s}\left(u_{i}^{*}-c u_{i+1}^{*}\right) d t, \forall s \in\left(0,\left|B_{R}\right|\right), \\
& -\frac{d \widehat{v}_{i+1}}{d s}(s)=\frac{s^{\frac{2}{n}-2}}{n^{2} \sigma_{n}^{2 / n}} \int_{0}^{s}\left(\widehat{v}_{i}-c \widehat{v}_{i+1}\right) d t, \forall s \in\left(0,\left|B_{R}\right|\right) .
\end{aligned}
$$

Thus for all $s \in\left(0,\left|B_{R}\right|\right)$, we have

$$
\frac{d \widehat{v}_{i+1}}{d s}(s)-\frac{d u_{i+1}^{*}}{d s}(s)-\frac{s^{\frac{2}{n}-2}}{n^{2} \sigma_{n}^{2 / n}} \int_{0}^{s}\left(c \widehat{v}_{i+1}-c u_{i+1}^{*}\right) d t \leq \frac{s^{\frac{2}{n}-2}}{n^{2} \sigma_{n}^{2 / n}} \int_{0}^{s}\left(u_{i}^{*}-\widehat{v}_{i}\right) d t .
$$

Thanks to the induction hypotheses, we get that

$$
\int_{0}^{s}\left(u_{i}^{*}-\widehat{v}_{i}\right) d t \leq 0, \forall s \in\left(0,\left|B_{R}\right|\right)
$$

and then

$$
\frac{d \widehat{v}_{i+1}}{d s}(s)-\frac{d u_{i+1}^{*}}{d s}(s)-\frac{s^{\frac{2}{n}-2}}{n^{2} \sigma_{n}^{2 / n}} \int_{0}^{s}\left(c \widehat{v}_{i+1}-c u_{i+1}^{*}\right) d t \leq 0
$$


Setting

$$
y(s)=\int_{0}^{s}\left(\widehat{v}_{i+1}-u_{i+1}^{*}\right) d t \quad \forall s \in\left(0,\left|B_{R}\right|\right)
$$

we get

$$
\left\{\begin{array}{c}
y^{\prime \prime}-\frac{c s^{\frac{2}{n}-2}}{n^{2} \sigma_{n}^{2 / n}} y \leq 0, \forall s \in\left(0,\left|B_{R}\right|\right) \\
y(0)=y^{\prime}\left(\left|B_{R}\right|\right)=0
\end{array} .\right.
$$

By maximum principle, we have that $y \geq 0$ which is what we need.

From the above proposition, we have the following corollary:

Corollary 2.1. Let $u, v$ be weak solutions of the polyharmonic problems (2.5) and (2.6) respectively. Then for every convex nondecreasing function $\phi:[0,+\infty) \rightarrow$ $[0,+\infty)$ we have

$$
\int_{B_{r}} \phi(|u|) d x \leq \int_{B_{r}} \phi(|v|) d x .
$$

Now, we state the following known result from $[12,25]$ :

Lemma 2.1. Let $f(s), g(s)$ be measurable, positive functions such that

$$
\int_{[0, r]} f(s) d s \leq \int_{[0, r]} g(s) d s, r \in[0, R] ;
$$

if $h(s) \geq 0$ is a decreasing function then

$$
\int_{[0, r]} f(s) h(s) d s \leq \int_{[0, r]} g(s) h(s) d s, r \in[0, R] .
$$

Then we have the following:

Proposition 2.4. Let $u, v$ be weak solutions of (2.5) and (2.6) respectively. For every convex nondecreasing function $\phi:[0,+\infty) \rightarrow[0,+\infty)$ we have

$$
\int_{B_{R}} \frac{\phi(|u|)}{|x|^{\alpha}} d x \leq \int_{B_{R}} \frac{\phi(|v|)}{|x|^{\alpha}} d x, 0 \leq \alpha<n .
$$

Next, we provide a Radial Lemma which will be used in the proof of Theorem 1.2. See $[14,24,29,47,52]$ :

LEMma 2.2. If $u \in W_{\text {rad }}^{1, \frac{n}{m}}\left(\mathbb{R}^{n}\right)$ then

$$
|u(x)| \leq\left(\frac{1}{m \sigma_{n}}\right)^{\frac{m}{n}} \frac{1}{|x|^{\frac{n-1}{n} m}}\|u\|_{W^{1, \frac{n}{m}}}
$$

for a.e. $x \in \mathbb{R}^{n}$. 
3. Proof of Theorem 1.1: Sharp inequality of exponential type for fractional integrals. We begin with proving the following result that is a modified version of the key lemma used to prove the Adams inequality in [2]:

Lemma 3.1. Let $0<\alpha \leq 1,1<p<\infty$ and $a(s, t)$ be a non-negative measurable function on $(-\infty, \infty) \times[0, \infty)$ such that (a.e.)

$$
\begin{aligned}
a(s, t) & \leq 1, \text { when } 0<s<t, \\
\sup _{t>0}\left(\int_{-\infty}^{0}+\int_{t}^{\infty} a(s, t)^{p^{\prime}} d s\right)^{1 / p^{\prime}} & =b<\infty .
\end{aligned}
$$

Then there is a constant $c_{0}=c_{0}(p, b, \alpha)$ such that if for $\phi \geq 0$,

$$
\int_{-\infty}^{\infty} \phi(s)^{p} d s \leq 1
$$

then

$$
\int_{0}^{\infty} e^{-F_{\alpha}(t)} d t \leq c_{0}
$$

where

$$
F_{\alpha}(t)=\alpha t-\alpha\left(\int_{-\infty}^{\infty} a(s, t) \phi(s) d s\right)^{p^{\prime}} .
$$

We sketch a proof here.

Proof. First, we have

$$
\int_{0}^{\infty} e^{-F_{\alpha}(t)} d t=\int_{-\infty}^{\infty}\left|E_{\lambda}\right| e^{-\lambda} d \lambda
$$

where $E_{\lambda}=\left\{t \geq 0: F_{\alpha}(t) \leq \lambda\right\}$.

We will separate the proof into two steps.

Step 1. There is a constant $c=c(p, b, \alpha)>0$ such that $F_{\alpha}(t) \geq-c$ for all $t \geq 0$.

Indeed, we will show that if $E_{\alpha \lambda} \neq \emptyset$, then $\lambda \geq-c$, and furthermore that if $t \in E_{\alpha \lambda}$, then

$$
\left(b^{p^{\prime}}+t\right)^{1 / p}\left(\int_{t}^{\infty} \phi(s)^{p} d s\right)^{1 / p} \leq A_{1}+B_{1}|\lambda|^{1 / p} .
$$

In fact, if $E_{\alpha \lambda} \neq \emptyset$ and $t \in E_{\alpha \lambda}$, then

$$
\begin{aligned}
t-\lambda & \leq t-\frac{F_{\alpha}(t)}{\alpha} \\
& \leq\left(\int_{-\infty}^{\infty} a(s, t) \phi(s) d s\right)^{p^{\prime}}
\end{aligned}
$$


Repeating the argument as in the proof of Lemma 1 in [2], we then have completed Step 1.

Step 2. $\left|E_{\lambda}\right| \leq A|\lambda|+B$, for constants $A$ and $B$ depending only on $p, b$ and $\alpha$. The proof of Step 2 is very similar to that in [2].

Hence, we finish the proof of the Lemma.

Using the above lemma, we now can prove Theorem 1.1.

Proof of Theorem 1.1. Set $u(x)=I_{n / p} * f(x)$, for $f \geq 0$. Using the notations

$$
\begin{aligned}
g(x) & =|x|^{\gamma-n}, \\
u^{* *}(t) & =\frac{1}{t} \int_{0}^{t} u^{*}(s) d s,
\end{aligned}
$$

we have by O'Neil's lemma that

$$
\begin{aligned}
u^{*}(t) & \leq u^{* *}(t) \\
& \leq t f^{* *}(t) g^{* *}(t)+\int_{t}^{\infty} f^{*}(s) g^{*}(s) d s \\
& =\left(\frac{\omega_{n-1}}{n}\right)^{1 / p^{\prime}}\left(p t^{-1 / p^{\prime}} \int_{0}^{t} f^{*}(s) d s+\int_{t}^{|\Omega|} f^{*}(s) s^{-1 / p^{\prime}} d s\right) .
\end{aligned}
$$

Now, we change variables by setting

$$
\phi(s)=|\Omega|^{1 / p} f^{*}\left(|\Omega| e^{-s}\right) e^{-s / p},
$$

then it can be checked that

$$
\begin{aligned}
\int_{\Omega} f(x)^{p} d x & =\int_{t}^{|\Omega|} f^{*}(t)^{p} d t \\
& =\int_{0}^{\infty} \phi(s)^{p} d s .
\end{aligned}
$$

By the Hardy-Littlewood inequality, note that with $h(x)=\frac{1}{|x|^{\alpha}}$, then $h^{*}(t)=$ $\left(\frac{\sigma_{n}}{t}\right)^{\frac{\alpha}{n}}$, we have

$$
\int_{\Omega} \frac{\exp \left(\left(1-\frac{\alpha}{n}\right) \frac{n}{\omega_{n-1}}|u(x)|^{p^{\prime}}\right)}{|x|^{\alpha}} d x \leq \sigma_{n}^{\frac{\alpha}{n}} \int_{0}^{|\Omega|} \frac{e^{\left(1-\frac{\alpha}{n}\right) \frac{n}{\omega_{n-1}} u^{*}(t)^{p^{\prime}}}}{t^{\frac{\alpha}{n}}} .
$$

Now, we do the change of variable

$$
t=|\Omega| e^{-s}
$$

Noting that

$$
d t=-|\Omega| e^{-s} d s,
$$


we get

$$
\begin{aligned}
& \sigma_{n}^{\frac{\alpha}{n}} \int_{0}^{|\Omega|} \frac{e^{\left(1-\frac{\alpha}{n}\right) \frac{n}{\omega_{n-1}} u^{*}(t)^{p^{\prime}}}}{t^{\frac{\alpha}{n}}} d t \\
& =\sigma_{n}^{\frac{\alpha}{n}}|\Omega|^{1-\frac{\alpha}{n}} \int_{0}^{\infty} \exp \left[\left(1-\frac{\alpha}{n}\right) \frac{n}{\omega_{n-1}} u^{*}\left(|\Omega| e^{-s}\right)^{p^{\prime}}-\left(1-\frac{\alpha}{n}\right) s\right] d s \\
& \leq \sigma_{n}^{\frac{\alpha}{n}}|\Omega|^{1-\frac{\alpha}{n}} \times \\
& \int_{0}^{\infty} \exp \left[\left(1-\frac{\alpha}{n}\right)\left[p\left(|\Omega| e^{-s}\right)^{-\frac{1}{p^{\prime}}} \int_{0}^{|\Omega| e^{-s}} f^{*}(z) d z+\int_{0}^{|\Omega|} f^{*}(z) z^{-\frac{1}{p^{\prime}}} d z\right)^{p^{\prime}}-\left(1-\frac{\alpha}{n}\right) s\right] d s \\
& =\sigma_{n}^{\frac{\alpha}{n}}|\Omega|^{1-\frac{\alpha}{n}} \int_{0}^{\infty} \exp \left[\left(1-\frac{\alpha}{n}\right)\left(p e^{s / p^{\prime}} \int_{s}^{\infty} \phi(w) e^{-\frac{w}{p^{\prime}}} d w+\int_{0}^{p^{\prime}} \phi(w)\right)^{-}-\left(1-\frac{\alpha}{n}\right) s\right] d s \\
& =\sigma_{n}^{\frac{\alpha}{n}}|\Omega|^{1-\frac{\alpha}{n}} \int_{0}^{\infty} \exp \left[-F_{\left(1-\frac{\alpha}{n}\right)}(s)\right] d s,
\end{aligned}
$$

where $F_{\left(1-\frac{\alpha}{n}\right)}(s)$ is as in Lemma 3.1 with

$$
a(s, t)=\left\{\begin{array}{c}
1 \text { for } 0<s<t \\
p e^{(t-s) / p^{\prime}} \text { for } t<s<\infty \\
0 \text { for }-\infty<s \leq 0
\end{array}\right.
$$

Thus it suffices to prove that

$$
\int_{0}^{\infty} \phi(s)^{p} d s \leq 1 \text { implies } \int_{0}^{\infty} \exp \left[-F_{\left(1-\frac{\alpha}{n}\right)}(s)\right] d s \leq c_{0},
$$

but this follows from Lemma 3.1 immediately.

4. Proof of Theorem 1.2: A singular Adams inequality on bounded domains. First, we will prove that

$$
\sup _{u \in W_{0}^{m, \frac{n}{m}}(\Omega),\left\|\nabla^{m} u\right\|_{\frac{n}{m} \leq 1}} \int_{\Omega} \frac{e^{\beta_{\alpha, n, m}|u|^{\frac{n}{n-m}}}}{|x|^{\alpha}} d x<\infty .
$$

To do that, it suffices to dominate an arbitrary $C^{m}$ function with compact support by a Riesz potential in such a way that the constants are precise. This can be done as in [2] through the following lemma:

Lemma 4.1. Let $u \in C_{0}^{\infty}\left(\mathbb{R}^{n}\right)$. Set $p=\frac{n}{m}$ and $p^{\prime}=\frac{n}{n-m}$. Then if $m$ is an odd positive integer,

$$
u(x)=(-1)^{\frac{m-1}{2}}\left(\frac{\omega_{n-1} \beta(n, m)}{n}\right)^{-1 / p^{\prime}} \times \int_{\mathbb{R}^{n}}|x-y|^{m-1-n}(x-y) \cdot \nabla^{m} u(y) d y
$$


and for $m$ an even positive integer

$$
u(x)=(-1)^{\frac{m}{2}}\left(\frac{\omega_{n-1} \beta(n, m)}{n}\right)^{-1 / p^{\prime}} \times \int_{\mathbb{R}^{n}}|x-y|^{m-n} \nabla^{m} u(y) d y .
$$

Proof of Theorem 1.2. It is clear that from Lemma 4.1, we have $\left(\frac{\omega_{n-1}}{n}\right) \beta(n, m)|u(x)|^{p^{\prime}} \leq\left[I_{m} *\left|\nabla^{m} u\right|(x)\right]^{p^{\prime}}$ and then we apply Theorem 1.1. This proves the first part of Theorem 1.2.

To show the second part of Theorem 1.2, suppose that $m$ is even: $m=2 k, k \in \mathbb{N}$, we will prove that

$$
\sup _{u \in W_{N}^{m, \frac{n}{m}}(\Omega),\left\|\nabla^{m} u\right\|_{\frac{n}{m} \leq 1}} \int_{\Omega} \frac{e^{\beta_{\alpha, n, m}|u|^{\frac{n}{n-m}}}}{|x|^{\alpha}} d x<\infty .
$$

By a density argument, it is enough to prove that

$$
\sup _{u \in C_{N}^{\infty}(\Omega),\left\|\nabla^{m} u\right\|_{\frac{n}{m}} \leq 1} \int_{\Omega} \frac{e^{\beta_{\alpha, n, m}|u|^{\frac{n}{n-m}}}}{|x|^{\alpha}} d x<\infty
$$

where

$$
C_{N}^{\infty}(\Omega)=\left\{u \in C^{\infty}(\Omega) \cap C^{m-2}(\bar{\Omega}):\left.u\right|_{\partial \Omega}=\left.\Delta^{j} u\right|_{\partial \Omega}=0,1 \leq j \leq\left[\frac{m-1}{2}\right]\right\} .
$$

Let $u \in C_{N}^{\infty}(\Omega)$ be such that $\left\|\nabla^{m} u\right\|_{\frac{n}{m}}=\left\|\Delta^{k} u\right\|_{\frac{n}{m}} \leq 1$ and set $f:=\Delta^{k} u$ in $\Omega$. Then $u$ is a solution of the Navier boundary value problem

$$
\left\{\begin{array}{c}
\Delta^{k} u=f \text { in } \Omega \\
u=\Delta^{j} u=0 \text { on } \partial \Omega, j \in\{1 \leq j<k\}
\end{array} .\right.
$$

Now, we extend $f$ by zero outside $\Omega$

$$
\bar{f}(x)=\left\{\begin{array}{c}
f(x), x \in \Omega \\
0, x \in \mathbb{R}^{n} \backslash \Omega
\end{array} .\right.
$$

Define

$$
\bar{u}=\left(\frac{n}{\omega_{n-1} \beta(n, m)}\right)^{\frac{n-m}{n}} I_{m} *|\bar{f}| \text { in } \mathbb{R}^{n},
$$

so that we have $(-1)^{k} \Delta^{k} u=|\bar{f}|$ in $\mathbb{R}^{n}$. It's clear that $\bar{u} \geq 0$ in $\mathbb{R}^{n}$ and

$$
\beta(n, m)|\bar{u}|^{\frac{n}{n-m}} \leq \frac{n}{\omega_{n-1}}\left(\frac{I_{m} *|\bar{f}|}{\|f\|_{\frac{n}{m}}}\right)^{\frac{n}{n-m}} \text { in } \mathbb{R}^{n} .
$$

It can be proved that $\bar{u} \geq|u|$ (see [47]) and then

$$
\begin{aligned}
\int_{\Omega} \frac{e^{\beta_{\alpha, n, m}|u|^{\frac{n}{n-m}}}}{|x|^{\alpha}} d x & \leq \int_{\Omega} \frac{e^{\beta_{\alpha, n, m}|\bar{u}|^{\frac{n}{n-m}}}}{|x|^{\alpha}} d x \\
& \leq \int_{\Omega} \frac{\exp \left(\left(1-\frac{\alpha}{n}\right) \frac{n}{\omega_{n-1}} \mid \frac{I_{\beta} * \bar{f}(x)}{\|\left.\left.\bar{f}\right|_{\frac{n}{m}}\right|^{\frac{n}{n-m}}}\right)}{|x|^{\alpha}} d x .
\end{aligned}
$$


By Theorem 1.1, (4.2) follows.

Moreover, it can be checked that the sequence of test functions which gives the sharpness of Adams' inequality in bounded domains [2] also gives the sharpness of $\beta_{\alpha, n, m}$. This completes the proof of Theorem 1.2.

\section{Proof of Theorem 1.3 and Theorem 1.4.}

\subsection{Proof of Theorem 1.3.}

Proof. Suppose that $m=2 k, k \in \mathbb{N}$. Let $u \in W^{m, \frac{n}{m}}\left(\mathbb{R}^{n}\right),\left\|(-\Delta+I)^{k} u\right\|_{\frac{n}{m}} \leq 1$, by the density of $C_{0}^{\infty}\left(\mathbb{R}^{n}\right)$ in $W^{m, \frac{n}{m}}\left(\mathbb{R}^{n}\right)$, without loss of generality, we can find a sequence of functions $u_{l} \in C_{0}^{\infty}\left(\mathbb{R}^{n}\right)$ such that $u_{l} \rightarrow u$ in $W^{m, \frac{n}{m}}\left(\mathbb{R}^{n}\right)$ and $\int_{\mathbb{R}^{n}}\left|(-\Delta+I)^{k} u_{l}\right|^{\frac{n}{m}} d x \leq 1$ and suppose that $\operatorname{supp} u_{l} \subset B_{R_{l}}$ for any fixed $l$. Let $f_{l}:=(-\Delta+I)^{k} u_{l}$, then supp $f_{l} \subset B_{R_{l}}$. Consider the problem

$$
\left\{\begin{array}{c}
(-\Delta+I)^{k} v_{l}=f_{l}^{\#} \text { in } B_{R_{l}} \\
v_{l} \in W_{N}^{m, 2}\left(B_{R_{l}}\right)
\end{array} .\right.
$$

By the property of rearrangement, we have

$$
\int_{B_{R_{l}}}\left|(-\Delta+I)^{k} v_{l}\right|^{\frac{n}{m}} d x=\int_{B_{R_{l}}}\left|(-\Delta+I)^{k} u_{l}\right|^{\frac{n}{m}} d x \leq 1
$$

and by the Hardy-Littlewood inequality and Proposition 2.4, we get

$$
\int_{B_{R_{l}}} \frac{\phi\left(\beta_{\alpha, n, m}\left|u_{l}\right|^{\frac{n}{n-m}}\right)}{|x|^{\alpha}} d x \leq \int_{B_{R_{l}}} \frac{\phi\left(\beta_{\alpha, n, m} \mid u_{l}^{\left.\#\right|^{\frac{n}{n-m}}}\right)}{|x|^{\alpha}} d x \leq \int_{B_{R_{l}}} \frac{\phi\left(\beta_{\alpha, n, m}\left|v_{l}\right|^{\frac{n}{n-m}}\right)}{|x|^{\alpha}} d x
$$

Now, writing

$$
\begin{aligned}
\int_{B_{R_{l}}} \frac{\phi\left(\beta_{\alpha, n, m}\left|v_{l}\right|^{\frac{n}{n-m}}\right)}{|x|^{\alpha}} d x & \leq \int_{B_{R_{0}}} \frac{\phi\left(\beta_{\alpha, n, m}\left|v_{l}\right|^{\frac{n}{n-m}}\right)}{|x|^{\alpha}} d x+\int_{B_{R_{l}} \backslash B_{R_{0}}} \frac{\phi\left(\beta_{\alpha, n, m}\left|v_{l}\right|^{\frac{n}{n-m}}\right)}{|x|^{\alpha}} d x \\
& =I_{1}+I_{2}
\end{aligned}
$$

where $R_{0}$ is a constant, independent of $l$ and $v_{l}$, and will be chosen later. Then we will prove that both $I_{1}$ and $I_{2}$ are bounded uniformly by a constant which depends on $R_{0}$

Using Theorem 1.2, we can estimate $I_{1}$. Indeed, we just need to construct an auxiliary radial function $w_{l} \in W_{N}^{m, \frac{n}{m}}\left(B_{R_{0}}\right)$ with $\left\|\nabla^{m} w_{l}\right\|_{\frac{n}{m}} \leq 1$ which increases the integral we are interested in. Such a function was constructed in [47]. For the sake of completion, we give the detail here. For each $i \in\{1,2, \ldots, k-1\}$ we define

$$
g_{i}(|x|):=|x|^{m-2 i}, \forall x \in B_{R_{0}}
$$

so $g_{i} \in W_{\text {rad }}^{m, \frac{n}{m}}\left(B_{R_{0}}\right)$. Moreover,

$$
\Delta^{j} g_{i}(|x|)=\left\{\begin{array}{c}
c_{i}^{j}|x|^{m-2 i-2 j} \text { for } j \in\{1,2, \ldots k-i\} \\
0 \text { for } j \in\{k-i+1, \ldots, k\}
\end{array} \quad \forall x \in B_{R_{0}}\right.
$$

where

$$
c_{i}^{j}=\prod_{h=1}^{j}[n+m-2(h+i)][m-2(i+h-1)], \forall j \in\{1,2, \ldots k-i\} .
$$


Let

$$
z_{l}(|x|):=v_{l}(|x|)-\sum_{i=1}^{k-1} a_{i} g_{i}(|x|)-a_{k} \forall x \in B_{R_{0}}
$$

where

$$
\begin{aligned}
& a_{i}:=\frac{\Delta^{k-i} v_{l}\left(R_{0}\right)-\sum_{j=1}^{i-1} a_{j} \Delta^{k-i} g_{j}\left(R_{0}\right)}{\Delta^{k-i} g_{i}\left(R_{0}\right)}, \forall i \in\{1,2, \ldots k-1\}, \\
& a_{k}:=v_{l}\left(R_{0}\right)-\sum_{i=1}^{k-1} a_{i} g_{i}\left(R_{0}\right) .
\end{aligned}
$$

We can check that (see [47])

$$
\begin{aligned}
z_{l} & \in W_{N, r a d}^{m, \frac{n}{m}}\left(B_{R_{0}}\right), \\
\nabla^{m} v_{l} & =\nabla^{m} z_{l} \text { in } B_{R_{0}} .
\end{aligned}
$$

We have the following lemma whose proof can be found in [47].

Lemma 5.1. For $0<|x| \leq R_{0}$, there exist positive constants $d\left(m, n, R_{0}\right)$ depending only on $m, n, R_{0}$ and $c_{m, n}$ depending only on $m, n$ such that

$$
\begin{aligned}
\left|v_{l}(|x|)\right|^{\frac{n}{n-m}} & \leq\left|z_{l}(|x|)\right|^{\frac{n}{n-m}}\left(1+c_{m, n} \sum_{j=1}^{k-1} \frac{1}{R_{0}^{2 j \frac{n}{m}-1}}\left\|\Delta^{k-j} v_{l}\right\|_{W^{1, \frac{n}{m}}}^{\frac{n}{m}}+\frac{c_{m, n}}{R_{0}^{n-1}}\left\|v_{l}\right\|_{W^{1, \frac{n}{m}}}^{\frac{n}{m}}\right)^{\frac{n}{n-m}} \\
& +d\left(m, n, R_{0}\right) .
\end{aligned}
$$

Now, setting

$$
w_{l}(|x|):=z_{l}(|x|)\left(1+c_{m, n} \sum_{j=1}^{k-1} \frac{1}{R_{0}^{2 j \frac{n}{m}-1}}\left\|\Delta^{k-j} v_{l}\right\|_{W^{1, \frac{n}{m}}}^{\frac{n}{m}}+\frac{c_{m, n}}{R_{0}^{n-1}}\left\|v_{l}\right\|_{W^{1, \frac{n}{m}}}^{\frac{n}{m}}\right) .
$$

Since

$$
\begin{aligned}
z_{l} & \in W_{N, r a d}^{m, \frac{n}{m}}\left(B_{R_{0}}\right), \\
\nabla^{m} v_{l} & =\nabla^{m} z_{l} \text { in } B_{R_{0}} .
\end{aligned}
$$

we have

$$
w_{l} \in W_{N, \text { rad }}^{m, \frac{n}{m}}\left(B_{R_{0}}\right)
$$

and

$$
\left\|\nabla^{m} w_{l}\right\|_{\frac{n}{m}}=\left\|\nabla^{m} z_{l}\right\|_{\frac{n}{m}}\left(1+c_{m, n} \sum_{j=1}^{k-1} \frac{1}{R_{0}^{2 j \frac{n}{m}-1}}\left\|\Delta^{k-j} v_{l}\right\|_{W^{1, \frac{n}{m}}}^{\frac{n}{m}}+\frac{c_{m, n}}{R_{0}^{n-1}}\left\|v_{l}\right\|_{W^{1, \frac{n}{m}}}^{\frac{n}{m}}\right) .
$$


Note that

$$
\begin{aligned}
\left\|\nabla^{m} z_{l}\right\|_{\frac{n}{m}} & =\left\|\nabla^{m} v_{l}\right\|_{\frac{n}{m}} \\
& \leq\left(1-\sum_{j=1}^{k-1}\left\|\Delta^{k-j} v_{l}\right\|_{W^{1, \frac{n}{m}}}^{\frac{n}{m}}-\left\|v_{l}\right\|_{W^{1, \frac{n}{m}}}^{\frac{n}{m}}\right)^{m / n} \\
& \leq 1-\frac{m}{n} \sum_{j=1}^{k-1}\left\|\Delta^{k-j} v_{l}\right\|_{W^{1, \frac{n}{m}}}^{\frac{n}{m}}-\frac{m}{n}\left\|v_{l}\right\|_{W^{1, \frac{n}{m}}}^{\frac{n}{m}}
\end{aligned}
$$

we have

$$
\begin{aligned}
\left\|\nabla^{m} w_{l}\right\|_{\frac{n}{m}} & \leq\left(1-\frac{m}{n} \sum_{j=1}^{k-1}\left\|\Delta^{k-j} v_{l}\right\|_{W^{1, \frac{n}{m}}}^{\frac{n}{m}}-\frac{m}{n}\left\|v_{l}\right\|_{W^{1, \frac{n}{m}}}^{\frac{n}{m}}\right) \times \\
& \times\left(1+c_{m, n} \sum_{j=1}^{k-1} \frac{1}{R_{0}^{2 j \frac{n}{m}-1}}\left\|\Delta^{k-j} v_{l}\right\|_{W^{1, \frac{n}{m}}}^{\frac{n}{m}}+\frac{c_{m, n}}{R_{0}^{n-1}}\left\|v_{l}\right\|_{W^{1, \frac{n}{m}}}^{\frac{n}{m}}\right) \\
& \leq 1
\end{aligned}
$$

if we choose $R_{0}$ sufficiently large.

Finally, note that

$$
I_{1} \leq e^{\beta_{0} d\left(m, n, R_{0}\right)} \int_{B_{R_{0}}} \frac{e^{\beta_{0}\left|w_{l}\right|^{\frac{n}{n-m}}}}{|x|^{\alpha}} d x
$$

using Theorem 1.2, we can conclude that $I_{1}$ is bounded by a constant since $w_{l} \in$ $W_{N, \text { rad }}^{m, \frac{n}{m}}\left(B_{R_{0}}\right)$ and $\left\|\nabla^{m} w_{l}\right\|_{\frac{n}{m}} \leq 1$.

Now, we will estimate $I_{2}$. Note that

$$
\begin{aligned}
I_{2} & =\int_{B_{R_{l}} \backslash B_{R_{0}}} \frac{\phi\left(\beta_{\alpha, n, m}\left|v_{l}\right|^{\frac{n}{n-m}}\right)}{|x|^{\alpha}} d x \\
& \leq \frac{1}{R_{0}^{\alpha}} \int_{B_{R_{l}} \backslash B_{R_{0}}} \phi\left(\beta_{\alpha, n, m}\left|v_{l}\right|^{\frac{n}{n-m}}\right) d x .
\end{aligned}
$$

By the same argument as that in [47], we can conclude that $I_{2} \leq c\left(m, n, R_{0}\right)$.

Combining the above estimates and using the Fatou lemma, we can conclude that

$$
\sup _{u \in W^{m, \frac{n}{m}}\left(\mathbb{R}^{n}\right),\left\|(-\Delta+I)^{\frac{m}{2}} u\right\|_{\frac{n}{m}} \leq 1} \int_{\mathbb{R}^{n}} \frac{\phi\left(\beta_{\alpha, n, m}|u|^{\frac{n}{n-m}}\right)}{|x|^{\alpha}} d x<\infty .
$$

When $\beta>\beta_{\alpha, n, m}$, again, it's easy to check that the sequence given by D. Adams [2] will make our supremum blow up. This completes the proof of Theorem 1.3. $\square$

\subsection{Proof of Theorem 1.4.}

Proof. It suffices to prove that

$$
\sup _{u \in W^{2,2}\left(\mathbb{R}^{4}\right), \int_{\mathbb{R}^{4}}\left(|\Delta u|^{2}+\tau|\nabla u|^{2}+\sigma|u|^{2}\right) \leq 1} \int_{\mathbb{R}^{4}} \frac{\left(e^{32 \pi^{2}\left(1-\frac{\alpha}{4}\right) u^{2}}-1\right)}{|x|^{\alpha}} d x<\infty .
$$


In fact, we will prove a stronger result that

$$
\sup _{u \in W^{2,2}\left(\mathbb{R}^{4}\right),\|-\Delta u+c u\|_{2} \leq 1} \int_{\mathbb{R}^{4}} \frac{\left(e^{32 \pi^{2}\left(1-\frac{\alpha}{4}\right) u^{2}}-1\right)}{|x|^{\alpha}} d x<\infty
$$

where $c>0$ is chosen such that $\|-\Delta u+c u\|_{2}^{2} \leq \int_{\mathbb{R}^{4}}\left(|\Delta u|^{2}+\tau|\nabla u|^{2}+\sigma|u|^{2}\right)$.

Let $u \in W^{2,2}\left(\mathbb{R}^{4}\right),\|-\Delta u+c u\|_{2} \leq 1$. By the density of $C_{0}^{\infty}\left(\mathbb{R}^{4}\right)$ in $W^{2,2}\left(\mathbb{R}^{4}\right)$, we can find a sequence of functions $u_{k}$ in $C_{0}^{\infty}\left(\mathbb{R}^{4}\right)$ such that $u_{k} \rightarrow u$ in $W^{2,2}\left(\mathbb{R}^{4}\right)$, supp $u_{k} \subset B_{R_{k}}$. Without loss of generality, we assume $\left\|-\Delta u_{k}+c u_{k}\right\|_{2} \leq 1$. By the Fatou lemma, we have

$$
\int_{\mathbb{R}^{4}} \frac{\left(e^{32 \pi^{2}\left(1-\frac{\alpha}{4}\right) u^{2}}-1\right)}{|x|^{\alpha}} d x \leq \liminf _{k \rightarrow \infty} \int_{B_{R_{k}}} \frac{\left(e^{32 \pi^{2}\left(1-\frac{\alpha}{4}\right) u_{k}^{2}}-1\right)}{|x|^{\alpha}} d x
$$

Now, set $f_{k}:=-\Delta u_{k}+c u_{k}$ and consider the problem

$$
\left\{\begin{array}{c}
-\Delta v_{k}+c v_{k}=f_{k}^{\#} \text { in } B_{R_{k}} \\
v_{k} \in W_{0}^{1,2}\left(B_{R_{k}}\right)
\end{array} .\right.
$$

We have that $v_{k} \in W_{N}^{2,2}\left(B_{R_{k}}\right)$. Moreover, by Proposition 2.4 and the property of rearrangement, we have

$$
\begin{gathered}
\left\|-\Delta u_{k}+c u_{k}\right\|_{2}=\left\|-\Delta v_{k}+c v_{k}\right\|_{2} \leq 1 \\
\int_{B_{R_{k}}} \frac{\left(e^{32 \pi^{2}\left(1-\frac{\alpha}{4}\right) u_{k}^{2}}-1\right)}{|x|^{\alpha}} d x \leq \int_{B_{R_{k}}} \frac{\left(e^{32 \pi^{2}\left(1-\frac{\alpha}{4}\right) v_{k}^{2}}-1\right)}{|x|^{\alpha}} d x .
\end{gathered}
$$

Now, we write

$$
\begin{aligned}
& \int_{B_{R_{k}}} \frac{\left(e^{32 \pi^{2}\left(1-\frac{\alpha}{4}\right) v_{k}^{2}}-1\right)}{|x|^{\alpha}} d x \\
= & \int_{B_{R_{0}}} \frac{\left(e^{32 \pi^{2}\left(1-\frac{\alpha}{4}\right) v_{k}^{2}}-1\right)}{|x|^{\alpha}} d x+\int_{B_{R_{k}} \backslash B_{R_{0}}} \frac{\left(e^{32 \pi^{2}\left(1-\frac{\alpha}{4}\right) v_{k}^{2}}-1\right)}{|x|^{\alpha}} d x \\
= & I_{1}+I_{2} .
\end{aligned}
$$

where $R_{0}$ only depends on $c$ and will be chosen later.

Choose $R_{0} \geq\left(\frac{1}{2 \pi^{2}}\left(\frac{1}{2 c}+\frac{1}{c^{2}}\right)\right)^{1 / 3}$, then by the Radial Lemma (Lemma 2.2) and 
(5.4), we have that $\left|v_{k}(x)\right| \leq 1$ when $|x| \geq R_{0}$. Thus

$$
\begin{aligned}
I_{2} & =\int_{B_{R_{k}} \backslash B_{R_{0}}} \frac{\left(e^{32 \pi^{2}\left(1-\frac{\alpha}{4}\right) v_{k}^{2}}-1\right)}{|x|^{\alpha}} d x \\
& \leq \frac{1}{R_{0}^{\alpha}} \int_{B_{R_{k}} \backslash B_{R_{0}}}\left(e^{32 \pi^{2}\left(1-\frac{\alpha}{4}\right) v_{k}^{2}}-1\right) d x \\
& \leq \frac{1}{R_{0}^{\alpha}} \sum_{j=1}^{\infty} \frac{\left(32 \pi^{2}\left(1-\frac{\alpha}{4}\right)\right)^{j}}{j !} \int_{B_{R_{k}}} v_{k}^{2} \\
& \leq \frac{1}{R_{0}^{\alpha}} \frac{1}{c^{2}} \sum_{j=1}^{\infty} \frac{\left(32 \pi^{2}\left(1-\frac{\alpha}{4}\right)\right)^{j}}{j !} \\
& =C(c) .
\end{aligned}
$$

Now, we estimate $I_{1}$. Put

$$
w_{k}(|x|)=\left\{\begin{array}{c}
v_{k}(|x|)-v_{k}\left(R_{0}\right), 0 \leq|x| \leq R_{0} \\
0, r \geq R_{0}
\end{array}\right.
$$

Then it's easy to check that $w_{k} \in W_{N}^{2,2}\left(B_{R_{0}}\right)$. Moreover, when $0<|x| \leq R_{0}$, using Radial Lemma 2.2, we have

$$
\begin{aligned}
\left(v_{k}(|x|)\right)^{2} & =\left[w_{k}(|x|)+v_{k}\left(R_{0}\right)\right]^{2} \\
& =w_{k}^{2}(|x|)+2 w_{k}(|x|) v_{k}\left(R_{0}\right)+\left[v_{k}\left(R_{0}\right)\right]^{2} \\
& \leq w_{k}^{2}(|x|)+w_{k}^{2}(|x|)\left[v_{k}\left(R_{0}\right)\right]^{2}+1+\left[v_{k}\left(R_{0}\right)\right]^{2} \\
& \leq w_{k}^{2}(|x|)\left[1+\frac{C}{R_{0}^{2}}\left\|v_{k}\right\|_{W^{1,2}}^{2}\right]+d\left(c, R_{0}\right) .
\end{aligned}
$$

Let

$$
z_{k}(|x|):=w_{k}(|x|)\left[1+\frac{C}{R_{0}^{2}}\left\|v_{k}\right\|_{W^{1,2}}^{2}\right]^{1 / 2}
$$

then $z_{k} \in W_{N}^{2,2}\left(B_{R_{0}}\right)$ since $w_{k} \in W_{N}^{2,2}\left(B_{R_{0}}\right)$. More importantly, we have

$$
\begin{aligned}
\left\|\Delta z_{k}\right\|_{2}^{2} & =\left\|\Delta w_{k}\right\|_{2}^{2}\left[1+\frac{C}{R_{0}^{2}}\left\|v_{k}\right\|_{W^{1,2}}^{2}\right] \\
& =\left\|\Delta v_{k}\right\|_{2}^{2}\left[1+\frac{C}{R_{0}^{2}}\left\|v_{k}\right\|_{W^{1,2}}^{2}\right] \\
& \leq\left(1-2 c\left\|\nabla v_{k}\right\|_{2}^{2}-c^{2}\left\|v_{k}\right\|_{2}^{2}\right)\left(1+\frac{C}{R_{0}^{2}}\left\|\nabla v_{k}\right\|_{2}^{2}+\frac{C}{R_{0}^{2}}\left\|v_{k}\right\|_{2}^{2}\right) \\
& \leq 1
\end{aligned}
$$

if we choose $R_{0}$ sufficiently large. 
Then using Theorem 1.2, we have

$$
\begin{aligned}
I_{1} & =\int_{B_{R_{0}}} \frac{\left(e^{32 \pi^{2}\left(1-\frac{\alpha}{4}\right) v_{k}^{2}}-1\right)}{|x|^{\alpha}} d x \\
& \leq C(c) \int_{B_{R_{0}}} \frac{e^{32 \pi^{2}\left(1-\frac{\alpha}{4}\right) z_{k}^{2}}}{|x|^{\alpha}} d x \\
& \leq C(c) .
\end{aligned}
$$

From (5.5) and (5.6), we get (5.2). Moreover, if we choose $0<c<\min \left\{\frac{\tau}{2}, \sqrt{\sigma}\right\}$, then we have

$$
\|-\Delta u+c u\|_{2}^{2} \leq \int_{\mathbb{R}^{4}}\left(|\Delta u|^{2}+\tau|\nabla u|^{2}+\sigma|u|^{2}\right), \forall u \in W^{2,2}\left(\mathbb{R}^{4}\right)
$$

and thus the proof of Theorem 1.4 is completed.

\section{REFERENCES}

[1] S. Adachi And K. TAnaka, Trudinger type inequalities in $R^{N}$ and their best exponents, Proc. of the Amer. Math. Soc., 128 (1999), pp. 2051-2057.

[2] D. R. Adams, A sharp inequality of J. Moser for higher order derivatives, Ann. of Math. (2), 128 (1988), no. 2, pp. 385-398.

[3] AdimurThi, Existence of positive solutions of the semilinear Dirichlet problem with critical growth for the $n$-Laplacian, Ann. Scuola Norm. Sup. Pisa Cl. Sci. (4), 17 (1990), no. 3, pp. 393-413.

[4] Adimurthi, N. Chaudhuri, and M. Ramaswamy, An improved Hardy-Sobolev inequality and its application, Proc. Amer. Math. Soc., 130 (2002), no. 2, pp. 489-505.

[5] Adimurthi and O. Druet, Blow-up analysis in dimension 2 and a sharp form of TrudingerMoser inequality, Comm. Partial Differential Equations, 29 (2004), pp. 295-322.

[6] Adimurthi, F. Robert, And M. Struwe, Concentration phenomena for Liouville's equation in dimension four, J. Eur. Math. Soc. (JEMS), 8 (2006), no. 2, pp. 171-180.

[7] Adimurthi And K. SAndeep, A singular Moser-Trudinger embedding and its applications, NoDEA Nonlinear Differential Equations Appl., 13 (2007), no. 5-6, pp. 585-603.

[8] Adimurthi AND S. L. YADAVA, Multiplicity results for semilinear elliptic equations in a bounded domain of $\mathbb{R}^{2}$ involving critical exponents, Ann. Scuola Norm. Sup. Pisa Cl. Sci. (4), 17 (1990), no. 4, pp. 481-504.

[9] Adimurthi And M. Struwe, Global compactness properties of semilinear elliptic equations with critical exponential growth, J. Funct. Anal., 175 (2000), no. 1, pp. 125-167.

[10] Adimurthi and K. Tintarev, On a version of Trudinger-Moser inequality with Möbius shift invariance, Calc. Var. Partial Differential Equations, 39 (2010), no. 1-2, pp. 203-212.

[11] Adimurthi And Y. YAng, An interpolation of Hardy inequality and Trudinger-Moser inequality in $\mathbb{R}^{N}$ and its applications, Int. Math. Res. Not. IMRN 2010, no. 13, pp. 2394-2426.

[12] A. Alvino, G. Trombetti, And P.-L. Lions, Comparison results for elliptic and parabolic equations via Schwarz symmetrization, Ann. Inst. H. Poincaré Anal. Non Linéaire, 7 (1990), no. 2, pp. 37-65.

[13] F. V. Atkinson And L. A. Peletier, Ground states and Dirichlet problems for $-\Delta u=f(u)$ in $\mathbb{R}^{2}$, Arch. Rational Mech. Anal., 96 (1986), no. 2, pp. 147-165.

[14] H. Berestycki And P.-L. Lions, Nonlinear scalar field equations. I. Existence of a ground state, Arch. Rational Mech. Anal., 82 (1983), no. 4, pp. 313-345.

[15] D. CAO, Nontrivial solution of semilinear elliptic equation with critical exponent in $R^{2}, \mathrm{Comm}$. Partial Differential Equations, 17 (1992), no. 3-4, pp. 407-435.

[16] L. Carleson And S. Y. A. Chang, On the existence of an extremal function for an inequality of J. Moser, Bull. Sci. Math. (2), 110 (1986), no. 2, pp. 113-127.

[17] A. Cianchi, Moser-Trudinger trace inequalities, Adv. Math., 217 (2008), no. 5, pp. $2005-2044$.

[18] A. Cianchi, Moser-Trudinger inequalities without boundary conditions and isoperimetric problems, Indiana Univ. Math. J., 54 (2005), no. 3, pp. 669-705.

[19] D. G. De Figueiredo, J. M. do Ó, And B. Ruf, On an inequality by N. Trudinger and J. Moser and related elliptic equations, Comm. Pure Appl. Math., 55 (2002), no. 2, pp. 135-152. 
[20] D. G. de Figueiredo, O. H. Miyagaki, And B. Ruf, Elliptic equations in $\mathbb{R}^{2}$ with nonlinearities in the critical growth range, Calc. Var. Partial Differential Equations, 3 (1995), no. 2, pp. $139-153$.

[21] J. M. Do Ó, Semilinear Dirichlet problems for the $N$-Laplacian in $\mathbb{R}^{N}$ with nonlinearities in the critical growth range, Differential Integral Equations, 9 (1996), no. 5, pp. 967-979.

[22] J. M. do Ó, E. MedeIros, ANd U. Severo, On a quasilinear nonhomogeneous elliptic equation with critical growth in $\mathbb{R}^{N}$, J. Differential Equations, 246 (2009), no. 4, pp. 1363-1386.

[23] L. Fontana and C. Morpurgo, Adams inequalities on measure spaces, Adv. Math., 226 (2011), no. 6, pp. 5066-5119.

[24] O. KAVIAN, Introduction à la théorie des points critiques et applications aux problèmes elliptiques, Springer-Verlag, Paris, 1993. viii+325 pp.

[25] S. Kesavan, Symmetrization \& applications, Series in Analysis, 3. World Scientific Publishing Co. Pte. Ltd., Hackensack, NJ, 2006. xii+148 pp.

[26] H. Kozono, T. SATo, And H. WAdAde, Upper bound of the best constant of a Trudinger-Moser inequality and its application to a Gagliardo-Nirenberg inequality, Indiana Univ. Math. J., 55 (2006), no. 6, pp. 1951-1974.

[27] N. LAM AND G. LU, Existence and multiplicity of solutions to equations of N-Laplacian type with critical exponential growth in $\mathbb{R}^{N}$, J. Funct. Anal., 262 (2012), no. 3, pp. 1132-1165.

[28] N. LAM AND G. LU, Existence of nontrivial solutions to Polyharmonic equations with subcritical and critical exponential growth, Discrete Contin. Dyn. Syst., 32 (2012), no.6, pp. 21872205 .

[29] N. LAm AND G. Lu, Sharp Adams type inequalities in Sobolev spaces $W^{m, \frac{n}{m}}\left(\mathbb{R}^{n}\right)$ for arbitrary interger $m$, J. Differential Equations, 253 (2012), pp. 1143-1171.

[30] N. LAM AND G. LU, Elliptic equations and systems with subcritical and critical exponential growth without the Ambrosetti- Rabinowitz condition, Journal of Geometric Analysis, DOI $10.1007 / \mathrm{s} 12220-012-9330-4$.

[31] N. LAm And G. LU, The Moser-Trudinger and Adams inequalities and elliptic and subelliptic equations with nonlinearity of exponential growth, in: Recent Development in Geometry and Analysis, Advanced Lectures in Mathematics, vol. 23, pp. 179-251.

[32] N. LAM AND G. LU, N-Laplacian equations in $\mathbb{R}^{N}$ with subcritical and critical growth without the Ambrosetti-Rabinowitz condition, Advanced Nonlinear Studies, in press.

[33] N. Lam And G. LU, A new approach to sharp Moser-Trudinger and Adams type inequalities: a rearrangement-free argument, preprint.

[34] N. LAm And G. Lu, Sharp Moser-Trudinger inequality on the Heisenberg group at the critical case and applications, Adv. Math., 231 (2012), no. 6, pp. 3259-3287.

[35] N. LAM AND G. Lu, On nonuniformly subelliptic equations of Q-sub-Laplacian type with critical growth in $\mathbb{H}^{n}$, Advanced Nonlinear Studies, 12 (2012), pp. 659-681.

[36] N. LAm, G. Lu, AND H. TANG, On sharp subcritical Moser-Trudinger inequality on the entire Heisenberg group and subelliptic PDEs, preprint.

[37] N. LAM, G. Lu, AND H. TANG, Sharp affine and improved Moser-Trudinger-Adams type inequalities, preprint.

[38] P. L. Lions, The concentration-compactness principle in the calculus of variations. The limit case. II, Rev. Mat. Iberoamericana, 1 (1985), no. 2, pp. 45-121.

[39] Y. X. Li And B. RuF, A sharp Trudinger-Moser type inequality for unbounded domains in $\mathbb{R}^{n}$, Indiana Univ. Math. J., 57 (2008), no. 1, pp. 451-480.

[40] G. LU AND Y. YANG, Adams' inequalities for bi-Laplacian and extremal functions in dimension four, Adv. Math., 220 (2009), pp. 1135-1170.

[41] G. Mancini And K. Sandeep, Moser-Trudinger inequality on conformal discs, Commun. Contemp. Math., 12 (2010), no. 6, pp. 1055-1068.

[42] J. Moser, A sharp form of an inequality by N. Trudinger, Indiana Univ. Math. J., 20 (1970/71), pp. 1077-1092.

[43] T. Ogawa And T. Ozawa, Trudinger type inequalities and uniqueness of weak solutions for the nonlinear Schrödinger mixed problem, J. Math. Anal. Appl., 155 (1991), no. 2, pp. 531-540.

[44] T. Ozawa, On critical cases of Sobolev's inequalities, J. Funct. Anal., 127 (1995), no. 2, pp. 259269.

[45] S. I. PohožAev, On the eigenfunctions of the equation $\Delta u+\lambda f(u)=0$ (Russian), Dokl. Akad. Nauk SSSR, 165(1965), pp. 36-39.

[46] B. RuF, A sharp Trudinger-Moser type inequality for unbounded domains in $\mathbb{R}^{2}$, J. Funct. Anal., 219 (2005), no. 2, pp. 340-367.

[47] B. Ruf And F. SAni, Sharp Adams-type inequalities in $\mathbb{R}^{n}$, to appear in Trans. Amer. Math. Soc.

[48] F. SANI, Exponential-type inequalities in $\mathbb{R}^{n}$ and applications to elliptic and biharmonic equa- 
tions, Ph.D. Thesis, 2011.

[49] M. Struwe, Critical points of embeddings of $H_{0}^{1, n}$ into Orlicz spaces, Ann. Inst. H. Poincaré Anal. Non Linéaire, 5 (1988), no. 5, pp. 425-464.

[50] M. StRuwe, Quantization for a fourth order equation with critical exponential growth, Math. Z., 256 (2007), no. 2, pp. 397-424.

[51] G. Talenti, Elliptic equations and rearrangements, Ann. Scuola Norm. Sup. Pisa Cl. Sci. (4), 3 (1976), no. 4, pp. 697-718.

[52] C. TARSI, Adams' Inequality and Limiting Sobolev Embeddings into Zygmund Spaces, Potential Anal., doi:10.1007/s11118-011-9259-4.

[53] G. J. Tian and X. J. Wang, Moser-Trudinger type inequalities for the Hessian equation, J. Funct. Anal., 259 (2010), no. 8, pp. 1974-2002.

[54] G. Trombetti AND J. L. VÁzQuez, A symmetrization result for elliptic equations with lowerorder terms, Ann. Fac. Sci. Toulouse Math. (5), 7 (1985), no. 2, pp. 137-150.

[55] N. S. Trudinger, On imbeddings into Orlicz spaces and some applications, J. Math. Mech., 17 (1967), pp. 473-483.

[56] G. Wang and D. Ye, A Hardy-Moser-Trudinger inequality, Adv. Math., 230 (2012), no. 1, pp. 294-320.

[57] V. I. Judovič, Some estimates connected with integral operators and with solutions of elliptic equations (Russian), Dokl. Akad. Nauk SSSR, 138 (1961), pp. 805-808.

[58] Y. YANG, Adams type inequalities and related elliptic partial differential equations in dimension four, J. Differential Equations, 252 (2012), no. 3, pp. 2266-2295. 